\title{
Southern and equatorial irregular variables
}

\section{Photoelectric photometry ${ }^{\star, \star \star}$}

\author{
D. Cieslinski ${ }^{1}$, F.J. Jablonski ${ }^{2,1}$, and J.E. Steiner ${ }^{3}$ \\ 1 Divisão de Astrofísica, Instituto Nacional de Pesquisas Espaciais, CP 515, 12201-970 São José dos Campos, Brazil \\ 2 Laboratório Nacional de Astrofísica-LNA/CNPq, CP 21, 37500-000 Itajubá, Brazil \\ 3 Instituto Astronômico e Geofísico, Universidade de São Paulo, CP 9638, 01065-970 São Paulo, Brazil
}

Received June 11; accepted October 10, 1996

\begin{abstract}
We present the results of an optical photometric survey of 616 irregular variables. The targets were selected among objects classified as L, L:, I, I:, IS and IS: in the General Catalogue of Variable Stars and New Catalogue of Suspected Variable Stars.
\end{abstract}

Key words: stars: variable - surveys - stars: AGB, post-AGB

\section{Introduction}

Irregular variables form a heterogeneous class of objects displaying slow to fast photometric variations with no obvious periodicities. The objects classified as L-type (also called slow irregular variables) are usually late-type giants (LB) or supergiants (LC) for which the variability is associated with pulsation in their atmospheres, while the variables designed as I are eruptives and the variability is caused by violent processes like flares that take place in their chromospheres or coronae. In addition, the I variables may be associated to nebulosity (IN) or may present rapid variability (IS).

Often, when a star is known to be variable, but no periodicity is found and no other information is available, it is classified as "irregular". This is common in the case of the stars found to be variable in photographic patrols. More detailed studies may show a large fraction of misclassification among these objects. In fact, several classes of variables can display irregular photometric variability without being a "classical" irregular variable of type L or

Send offprint requests to: D. Cieslinski, deo@das.inpe.br

* Based on observations made at Laboratório Nacional de Astrofísica/CNPq, Brazil.

** Table 1 only available in electronic form at CDS via ftp cdsarc.u-strasbg.fr (130.79.128.5) or via http://cdsweb.ustrasbg.fr/Abstract.html
I as defined in the General Catalogue of Variable Stars (Kukarkin et al. 1969). Cataclysmic variables and QSOs are good examples. In the past, a number of active galactic nuclei were initially classified as irregular variables, like the radio galaxy 3C120 (Penston 1968); the prototype of the "blazars", BL Lac (Schmitt 1968); QSOs like V395 Her (Bond 1972). This has also happened with peculiar binary stars, like the X-ray binary HZ Her (Davidsen et al. 1972, and references therein) and the intermediate polar V1223 Sgr (Steiner et al. 1981). Systematic searches for nebulosities led to the discovery of extragalactic objects among previously classified irregular variables (Bond 1973). Systematic observations using $U B V$ photoelectric photometry should be efficient in selecting peculiar galactic objects as well. This motivated us to do a photometric and spectroscopic study on irregular variables.

In this paper we present the results from photoelectric photometry of 616 stars. Follow-up spectroscopic observations (Part II) and data analysis (Part III) will be published elsewhere. A preliminary report on this project was published by Steiner et al. (1988).

\section{Observations and data reduction}

The target stars observed in the present program were selected among the objects classified as L, L:, I, I:, IS and IS: in the General Catalogue of Variable StarsGCVS (Kukarkin et al. 1969 and later supplements; Kholopov et al. 1985, 1987) and in the New Catalogue of Suspected Variable Stars-NSV (Kukarkin et al. 1982). Objects with $\delta<+10^{\circ}$ were chosen.

The observations were carried out from 1985 to 1994 with the 0.6-m Zeiss and 1.6-m Boller \& Chivens telescopes of the Laboratório Nacional de Astrofísica (LNA/CNPq), in southeast Brazil. The FOTEX and FOTRAP photometers were used. The former is a conventional one channel $U B V$ photometer equipped with a thermoelectrically cooled EMI 9789QA photomultiplier 
operating in the photon counting mode, while the second photometer uses a high-speed $(1200 \mathrm{rpm})$ rotating filter wheel which allows quasi-simultaneous photometry in the $U B V(R I)_{\mathrm{C}}$ bands. The detector used in this case is a thermoelectrically cooled Hamamatsu R943-02 photomultiplier.

Integration times of 5 to $20 \mathrm{~s}$ (depending on the brightness of the star) were used with the FOTEX photometer. Usually, 5 readings on the star and 5 on the sky were done with diaphragms of $19^{\prime \prime}$ and $12^{\prime \prime}$ in diameter for the $0.6-\mathrm{m}$ and $1.6-\mathrm{m}$ telescopes, respectively. In the FOTRAP photometer the integration time can be set by selecting the number of rotation cycles of filter wheel (normally $50 \mathrm{cy}-$ cles, providing $19.8 \mathrm{~s}$ of integration per filter) or by setting a threshold to the observational error, normally $0.5 \%$ in the worst case, usually the $U$ filter. Diaphragms of the same size as used in the FOTEX were also used in this photometer.

Photometric sequences of standard stars from Landolt (1973) or Graham (1982) were observed each night in order to correct for the effects of atmospheric extinction and to transform the observations to the standard systems. For a few nights in which we did not have calibration sequences we used transformation coefficients determined in the same mission being, in most cases, calibrations from previous or subsequent nights. The procedures that we used for the reduction of the data are those described in Jablonski et al. (1994). This reference also contain a detailed description of the FOTRAP photometer and the photometric system.

Observations in the $U$ filter with the FOTEX photometer were made, preferentially, for stars bluer than $B-V=1.4$ and for some of the brightest red stars. For many of the blue and bluish objects more than one measurement was done. In this case, we are interested in search for variabilities with time scales from several hours to few days.

The $U B V(R I)_{\mathrm{C}}$ data obtained are shown in Cols. 3 to
7 in Table 1 (this table is only available in electronic form, see footnote at the beginning of the paper). The associated uncertainties (Cols. 8 to 12) take into account the errors due to photon noise in the measurements and errors associated with the transformation to standard system. Column 2 shows the time of the middle of the observation in heliocentric Julian day. $\mathrm{y}$

Acknowledgements. We are grateful to the LNA staff for the observing assistance during the missions. We also thank the referee, R. Viotti, for his valuable comments and suggestions. D. Cieslinski acknowledges the support of CNPq (Conselho Nacional de Desenvolvimento Científico e Tecnológico) and FAPESP (Fundação de Amparo à Pesquisa do Estado de São Paulo), under grants 140587/89-6 and 85/1383-9.

\section{References}

Bond H.E., 1972, ApJL 174, 163

Bond H.E., 1973, ApJL 181, 23

Davidsen A., Henry J.P., Middleditch J., Smith H.E., 1972, ApJL 177, 97

Graham J.A., 1982, PASP 94, 244

Jablonski F.J., Baptista R., Barroso Jr.J., et al., 1994, PASP 106, 1172

Kholopov P.N., et al., 1985, 1987, General Catalogue of Variable Stars-GCVS4. Nauka Publishing House, Moscow

Kukarkin B.V., et al., 1969, General Catalogue of Variable Stars-GCVS3, Academy of Sciences of USSR, Moscow

Kukarkin B.V., et al., 1982, New Catalogue of Suspected Variable Stars-NSV. Nauka Publishing House, Moscow

Landolt A.U., 1973, AJ 78, 959

Penston M.V., 1968, IBVS No. 255

Schmitt J.L., 1968, Nat 218, 663

Steiner J.E., Schwartz D.A., Jablonski F.J., Busko I.C., Watson M.G., Pye J.P., McHardy I.M., 1981, ApJL 249, 21

Steiner J.E., Cieslinski D., Jablonski F.J., 1988, in: Progress and Opportunities in Southern Hemisphere Optical Astronomy, Blanco V.M. and Phillips M.M. (eds.), ASP Conf. Ser. 1, 67 